\title{
Research Note \\ IXAE observations of the X-ray pulsar XTE J1946+274
}

\author{
B. Paul $^{1}$, P. C. Agrawal ${ }^{1}$, K. Mukerjee ${ }^{1}$, A. R. Rao ${ }^{1}$, S. Seetha ${ }^{2}$, and K. Kasturirangan ${ }^{2}$ \\ 1 Tata Institute of Fundamental Research, Homi Bhabha Road, Mumbai 400 005, India \\ 2 ISRO Satellite Centre, Airport Road, Vimanpura PO Bangalore 560 017, India
}

Received 1 January 2001 / Accepted 26 February 2001

\begin{abstract}
We report results from two observations of the transient binary X-ray pulsar XTE J1946+274 with the Indian X-ray Astronomy Experiment (IXAE) made in September 18-30 1999 and June 28-July 7 2000. The pulsar has a $\sim 80$ days orbital period during which the X-ray intensity varies almost sinusoidally between intensity levels of 5 and 50 mCrab. The mid-time of the two observations with the IXAE are separated by an orbital phase of 0.56 and were in the decaying and rising parts of its orbital modulation, respectively. During both the observations, strong pulsations with $15.8 \mathrm{~s}$ period are detected in the 2-6 and 6-18 keV energy bands of IXAE. The pulse profiles in both the observations are double peaked and identical with a similar pulse fractions of $\sim 30 \%$ in the two energy bands. The pulse periods and its local derivatives are obtained from the IXAE observations. The known pulse period history shows small deviations from an otherwise nearly constant spin-up trend of the pulsar since its discovery. If the measured local period derivatives are a sum of a nearly constant spin-up of the pulsar and effect of its orbital motion, it suggests that the orbit of this Be X-ray binary is eccentric. The RXTE-ASM light curve shows a continuous detection of 10 orbital modulation of the source since its discovery.
\end{abstract}

Key words. X-rays: stars - pulsars: individual: XTE J1946+274

\section{Introduction}

The transient X-ray source XTE J1946+274 was discovered with the RXTE-ASM in 1998 September and immediate observations with the RXTE-PCA revealed a pulsar in it (Smith \& Takeshima 1998). Pulsations from the same source were also detected with the Burst and Transient Source Experiment (BATSE) onboard CGRO, which also found it to be spinning up (Wilson et al. 1998), a property that is commonly seen in transient sources with a pulsar. The pulse profile was reported to be double-peaked in the low energy band (3-20 keV) of RXTE-PCA and single-peaked in the hard X-ray band $(20-50 \mathrm{keV})$ of BATSE. The position of this source derived from scans with the RXTE-PCA suggested it to be the same source as 3A 1942+274 (Takeshima \& Chakrabarty 1998), but the association is not certain (Campana et al. 1999). In addition to the pulsation properties, Beppo-SAX observations revealed a complex continuum spectrum, with a weak iron emission line at $6.6 \mathrm{keV}$ and a column density of $1.610^{22} \mathrm{~cm}^{-2}$ (Campana et al. 1998). In the light curve obtained with the RXTE-ASM during the first year, there is an indication of a $\sim 80 \mathrm{~d}$ periodicity, probably

Send offprint requests to: B. Paul, e-mail: bpaul@tifr.res.in caused by the neutron star's motion along an eccentric orbit (Campana et al. 1999). The periodic X-ray modulation observed in XTE J1946+274 at the orbital period of the binary is known to be present in many other X-ray pulsars with a Be-star as companion.

On two occasions we made observations of this new pulsar with the Indian X-ray Astronomy Experiment (IXAE), once in 1999 September and again in 2000 JuneJuly, continuously for 13 and 10 days, respectively. From observations of this new transient X-ray binary pulsar, in addition to measurements of the pulse period and its derivative, we aimed to study the pulse profiles, their energy dependence, and if present, the changes of the profiles with luminosity. The latter gives us a clue about the changes in accretion geometry with the mass accretion rate. In the following sections we describe the instrument and the observations, analysis, and the results, followed by a discussion highlighting the properties of this relatively new and less studied X-ray pulsar.

\section{The IXAE and the observations}

The X-ray observations were made with the IXAE, which was launched aboard the Indian Remote Sensing SatelliteP3 (IRS-P3) using a Polar Satellite Launch Vehicle 


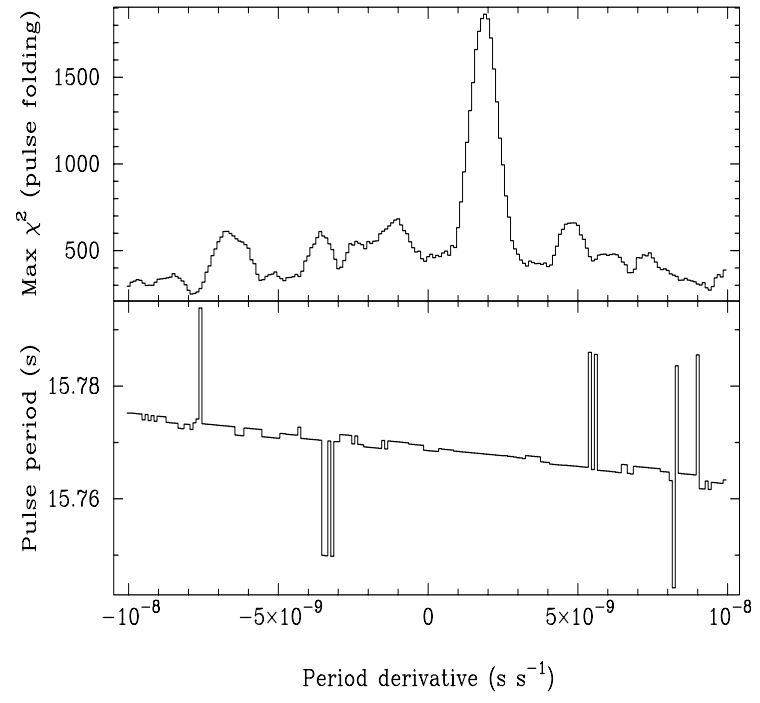

Fig. 1. Result of the epoch folding and $\chi^{2}$ maximisation with different pulse periods and period derivatives are shown here. See the text for details of the analysis technique used here. A few scattered points in the lower panel are for the cases where due to aliasing, a wrong period gave higher value of the $\chi^{2}$. This figure is for the observation made in 2000

(PSLV) on 1996 March 21. The satellite is in a circular orbit at an altitude of $830 \mathrm{~km}$ and inclination of $98^{\circ}$. The IXAE includes three collimated Pointed Proportional Counters (PPCs) with an effective area of about $1200 \mathrm{~cm}^{2}$ and field of view $2.3^{\circ} \times 2.3^{\circ}$. In the nominal mode of observation, light curves in two different energy bands of 2-6 and $6-18 \mathrm{keV}$ are available with different time resolutions. A more detailed description of the IXAE has been given by Rao et al. (1998). Since the satellite is in a polar orbit and most of the orbits pass through the South Atlantic Anomaly (SAA) region, useful data are obtained only when the satellite is in the latitude range of $50^{\circ} \mathrm{N}$ to $30^{\circ} \mathrm{S}$ and is also outside the SAA region. Usually in one day's observation, useful data is available for 5 consecutive orbits of the satellite, each for about $12-15 \mathrm{~min}$. The total background count rate in the $2-18 \mathrm{keV}$ energy band from the three detectors is about 40 counts per second.

Two observation campaigns were made, one during 1999 September 18-30 and another during 2000 June 28July 07 . All the observations were made in a mode in which light curves with $1 \mathrm{~s}$ time resolution are available in the two energy bands. Each day, a maximum of 5 stretches of $\sim 750$ s data were available. The total usable low background data in the 1999 and 2000 observations were for 30000 and $32000 \mathrm{~s}$, respectively. Of the three proportional counters that comprise the IXAE, only two were available during the second observation made in 2000, this reduced the effective area of the instruments to $800 \mathrm{~cm}^{2}$. We have also used the long term light curve of XTE J1946+274 in the $3-15 \mathrm{keV}$ band obtained with the RXTE-ASM to confirm the presence of $\sim 80$ day periodicity since it was last reported (Campana et al. 1999) and obtained an improved value of the period.
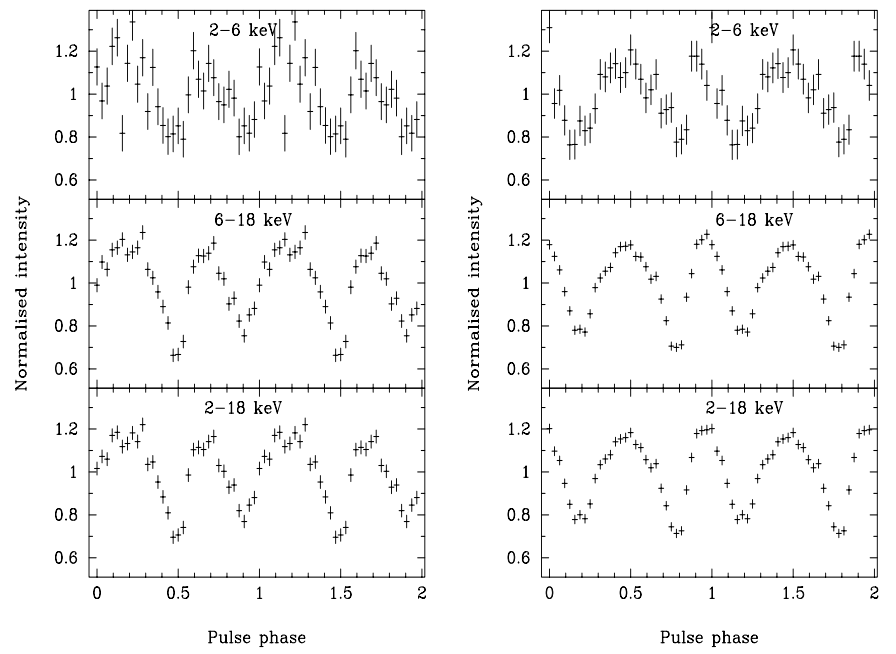

Fig. 2. The pulse profiles of XTE J1946+274 in the $2-6 \mathrm{keV}$, 6-18 keV and 2-18 keV bands observed with the IXAE during 1999 September and 2000 June-July are shown in the left and right columns, respectively. The pulse phases are arbitrarily chosen for the two observations

\section{Analysis and results}

Light curves obtained from the two PPCs with a time resolution of $1 \mathrm{~s}$ were first corrected to the solar system barycenter. A search for pulsation was carried out in the period range of $14-16 \mathrm{~s}$ by the pulse folding and $\chi^{2}$ maximising method. In addition to the main peak corresponding to the pulse period, aliasing due to a low duty cycle of the IXAE and a regular pattern in the light curve gives a few more significant peaks. The long term period history of this pulsar, including the initial RXTE, BATSE, and Beppo-SAX observations in 1998 and the recent pulse period measurements with BATSE that are publicly available, shows a nearly constant spin-up trend. The average rate of spin-up obtained from this history corresponds to a pulse decoherence time scale of $\sim 4$ days. The duration of both the IXAE observations are much longer than the decoherence time scale. We therefore carried out a pulsation analysis allowing for a period derivative in a large range that is a few times more than the average long term value.

The pulse folding and $\chi^{2}$ maximising method was applied with a period derivative, and the same was done for 400 different period derivatives in the range of $\dot{P}=$ $\pm 10^{-8} \mathrm{~s} \mathrm{~s}^{-1}$. From each trial, the maximum $\chi^{2}$ and the corresponding pulse period was selected. The resulting distribution of maximum $\chi^{2}$ and the corresponding pulse periods obtained from the second observation are plotted in Fig. 1 against the period derivatives with which the respective trials were carried out. From the first observation also, similar results were obtained. In the upper panel of Fig. 1, a gradual but definite increase in the maximum $\chi^{2}$ can be seen for $\dot{P}=1.8610^{-9} \mathrm{~s} \mathrm{~s}^{-1}$. This, therefore, allows us to calculate the pulse period and its derivative during each observation. 


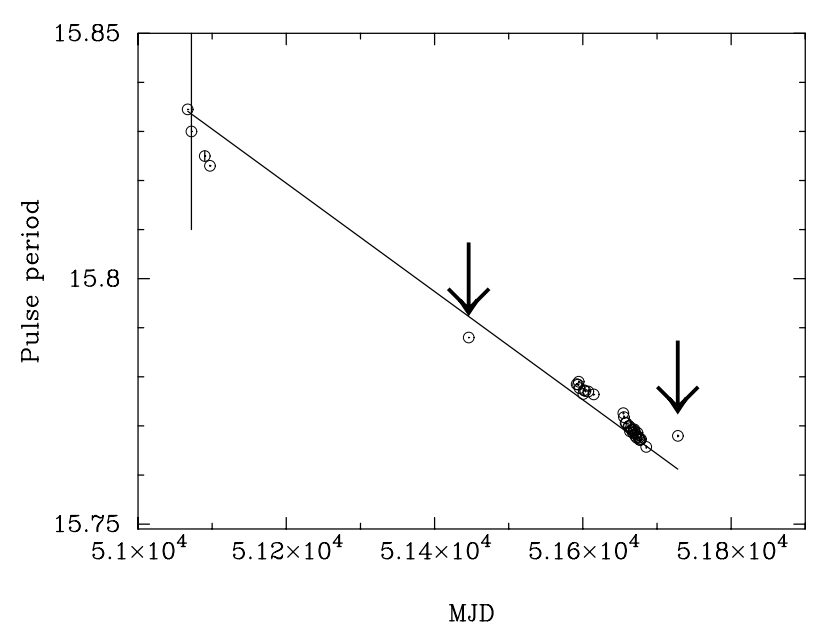

Fig. 3. The pulse period history of XTE J1946+274 including the earlier observations with RXTE-PCA, Beppo-SAX, and BATSE. The cluster of points near the end are from the publicly available recent observations with the BATSE. The two IXAE observations are marked with arrows. The straight line represents the best fitted straight line through the period history

We have also carried out an independent measurement of the pulse period and the period derivative during the two observations by pulse arrival time analysis. Using the $P$ and $\dot{P}$ values obtained from the above analysis, templates of the pulse profiles were generated for the two observations. Pulse profiles were generated from each day's observation and arrival times of the pulses for the midtime of each day's observation were obtained by crosscorrelating the pulse profiles with the respective pulse templates. A linear fit of the pulse number with the arrival times showed large systematic residuals. Introduction of a quadratic term improved the fit considerably, with no systematic trend in the residuals. The linear and quadratic terms give us the pulse periods and the period derivatives during the two observations. The results obtained from the two observations using the two above mentioned methods are identical and are given in Table 1.

Table 1. The pulse period and period derivatives determined from the IXAE observations

\begin{tabular}{|l|l|l|}
\hline Obs mid-time (MJD) & 51445.0 & 51727.5 \\
\hline$P(\mathrm{~s})$ & $15.78801(04)$ & $15.76796(18)$ \\
\hline$\dot{P}\left(10^{-9} \mathrm{~s} \mathrm{~s}^{-1}\right)$ & $-1.54(37)$ & $1.86(45)$ \\
\hline
\end{tabular}

The pulse profiles are shown in Fig. 2 for both the observations in the energy bands of $2-6,6-18$ and $2-18 \mathrm{keV}$. The profiles are double-peaked with a pulse fraction of about $20 \%$ and $30 \%$ in the $2-6$ and $6-18 \mathrm{keV}$ energy bands, respectively. The two peaks in the profile have widths of $60 \%$ and $40 \%$ of the pulse phase and identical amplitude. The minimum following the main peak is deeper than the other one. The pulse profiles are similar in shape in the two energy bands of $2-6 \mathrm{keV}$ and $6-18 \mathrm{keV}$.

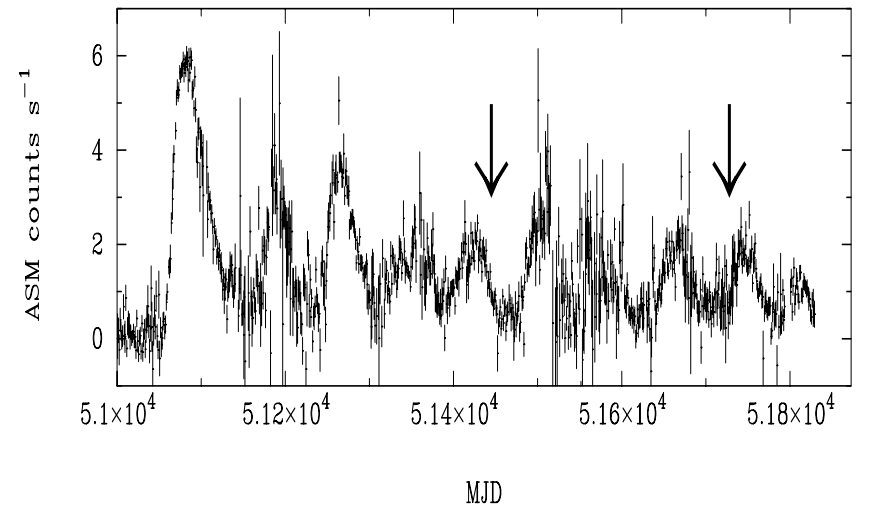

Fig. 4. The RXTE-ASM light curve of XTE J1946+274 showing the periodic intensity variations since its recent outburst in 1998. Epochs of the two IXAE observations are marked with arrows

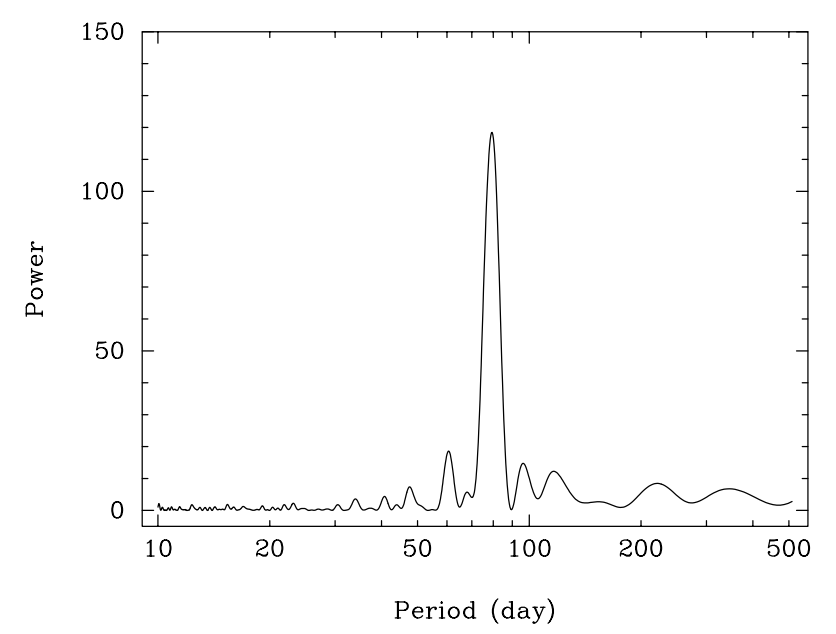

Fig. 5. The periodogram obtained from the RXTE-ASM data excluding the first flare

The pulse period history of XTE J1946+274 including the earlier published measurements, the publicly available recent measurement with BATSE, and the two IXAE measurements presented here are shown in Fig. 3. The period history shows a nearly constant spin-up with some local deviations. The average period derivative is obtained to be $\dot{P}=-1.2710^{-9} \mathrm{~s} \mathrm{~s}^{-1}$, and the trend is shown in Fig. 3 with a straight line.

The RXTE-ASM light curve of the source is shown in Fig. 4 in which the epochs of the two IXAE observations have also been marked. Since its outburst in September 1998, ten cycles of periodic modulations have been clearly seen. The amplitudes of the successive orbital modulations show an almost linear decay. As was noticed earlier (Campana et al. 1999), the first flare is not equally spaced, as are the other flares. First, we calculated periodgram from the data excluding the first flare and the result is shown in Fig. 5. The orbital period obtained from analysis of the recent RXTE-ASM light curve is $79.10 \pm 0.25 \mathrm{~d}$, which is slightly higher than the same obtained from the early RXTE-ASM light curve, $77.3 \pm 1.0 \mathrm{~d}$ (Campana et al. 1999). Though the periodogram does not show any 
peak at twice this period, some difference can be noticed between the odd and even flares by folding the light curve with two times the observed period. Inclusion of the first flare gives a still higher value for the orbital period $(82.1 \mathrm{~d}$ ) with somewhat less significance for the peak detection.

\section{Discussion}

The transient nature, orbital modulation and detection of a Be star in the X-ray error radius (see the references in Campana et al. 1999) suggests that XTE J1946+274 is a Be-star binary. The first and brightest of the outbursts seems to be a type II outburst, and the more regular and smaller subsequent periodic outbursts (see Fig. 4) seem to be of type I. As the source was not detected in the RXTE-ASM observations prior to September 1998, it can definitely be termed as a transient source. However, the prolonged and continued existence of the transient state for more than 10 orbital periods makes it different from most of the other transient Be-star binary pulsars. The common transient Be-star binary pulsars (Bildsten et al. 1997) are not known to have such a long outburst phase. There are some Be-star binary X-ray pulsars having steady X-ray emission with orbital modulation and occasional short transient episodes (e.g. 4U 1907+09, see Mukerjee et al. 2001). XTE J1946+274 appears to be very different in the nature of its outburst compared to most of the other pulsars with a Be-star as companion. In this regard, it should be noted that strong quasi-periodic oscillations in the frequency range of 10 to $400 \mathrm{mHz}$ have been detected in several transient X-ray pulsars, (see Paul \& Rao 1998; Mukerjee et al. 2001 and references therein). We have also generated the power density spectrum in the frequency range of 1 to $500 \mathrm{mHz}$, which does not show any significant feature apart from the signatures of the regular pulsations.

A fan or pencil type geometry of the beaming pattern, angles between the magnetic axis and the spin axis, and that between the observer line of sight and spin axis defines the pulse profiles of X-ray pulsars. In Fig. 2, we see that the intensity levels of the maxima or the minima are not the same. Although the two maxima are separated by a pulse phase of 0.5 , this is not so for the two minima. Such asymmetric features in the pulse profile indicates that the emission pattern in XTE J1946+274 is complex and it implies that the two magnetic poles may not be positioned diametrically opposite to each other.
Unfortunately, with the two IXAE observations lasting for 13 and 10 days, which cover only a small fraction of the orbital period, we are unable to derive the orbital parameters. The local period derivatives, measured from the present observations are the sum of the spin-up rate due to the accretion process and the effect of orbital motion. If we assume that the long term spin-up rate due to the accretion of matter is constant $\left(\dot{P}=-1.2710^{-9} \mathrm{~s} \mathrm{~s}^{-1}\right.$, Fig. 3), the period derivatives only due to the orbital motion are $\dot{P}=-2.710^{-10} \mathrm{~s} \mathrm{~s}^{-1}$ and $\dot{P}=3.1310^{-9} \mathrm{~s} \mathrm{~s}^{-1}$, respectively for the two observations separated by an orbital phase of 0.56 . In any circular orbit, the period derivative induced by orbital motion should have an identical absolute value for an orbital phase difference of 0.50 . In the present measurements, the absolute values differ by a factor of $\sim 10$, indicating a large eccentricity for this system. Given the spin and the orbital periods of this pulsar, Campana et al. (1999) also predicted significant eccentricity in this system. However, as some asymmetry is noticed in the odd and even flares, if the orbital period is twice that assumed here, the two IXAE observations are separated only by an orbital phase of 0.28 , and in that case the measured $\dot{P}$ values would be allowed, even in a circular geometry.

Acknowledgements. We thank K. Thyagrajan, Project Director of IRS-P3, R. N. Tyagi, Manager PMO, and J. D. Rao and his team at ISTRAC for their support. The valuable contributions of the technical and engineering staff of TIFR, ISAC and ISTRAC in making the IXAE payload and carrying out observations with it are gratefully acknowledged. We also thank the RXTE-ASM and BATSE pulsar teams for making their valuable data publicly available.

\section{References}

Bildsten, L., Chakrabarty, D., Chiu, J., et al. 1997, ApJS, 113, 367

Campana, S., Israel, G. L., Stella, L., \& Santangelo, A. 1998, IAU Circ., No. 7039

Campana, S., Israel, G. L., \& Stella, L. 1999, A\&A, 352, L91

Mukerjee, K., Agrawal, P. C., Paul, B., et al. 2001, ApJ, 548, 368

Paul, B., \& Rao, A. R. 1998, A\&A, 337, 815

Rao, A. R., Agrawal, P. C., Paul, B., et al. 1998, A\&A, 330, 181

Smith, D. A., \& Takeshima, T. 1998, IAU Circ., No. 7014

Takeshima, T., \& Chakrabarty, D. 1998, IAU Circ., No. 7016

Wilson, C. A., Finger, M. H., Wilson, R. B., \& Scott, D. M. 1998, IAU Circ., No. 7014 